\title{
Size Determination of Pollens Using Gravitational and Sedimentation Field-Flow Fractionation
}

\author{
Dong Young Kang, Min-Seok Son, Chul Hun Eum, ${ }^{+}$Won-Suk Kim, ${ }^{\ddagger}$ and Seungho Lee \\ Deparment of Chemistry, Hamam Lniversity, Daejeon 306-791, Korea. E-mail: sleethannamackr \\ - Korea Institute of Geoscience and Mineral Resources, Daejeon 305-350, Korea \\ ${ }^{\ddagger}$ Analutical Center, LG Chemicals, Daejeon 305-380, Korea \\ Received Jahuary 10, 2007
}

\begin{abstract}
Pollens are known to be an allergen. They penetrate human respiratory system. triggering a type of seasonal allergic rhinitis called pollen allergy (hey fever). The purpose of this study is to test two field-flow fractionation (FFF) techniques gravitational FFF (GrFFF) and sedimentation FFF (SdFFF), for their applicability to sizecharacterization of micron-sized pollens. Both GrFFF and SdFFF are elution techniques. providing sequential elution of particles based on size. They allow the size distribution as well as the mean size of the sample to be determined from the elution time. In this study. GrFFF and SdFFF were used to determine the size distribution of Paper Mulberry and Bermuda Grass pollens. For the Paper Mulberry pollen the mean size obtained by GrFFF is $12.7 \mu \mathrm{m}$. and agrees rather well with the $\mathrm{OM}$ data with the relative error of $8.0 \%$. For the Bermuda Grass pollen the mean size obtained by GrFFF is $32.6 \mu \mathrm{m}$ with the relative error of $12.3 \%$. The mean sizes determined by SdFFF are 12.4 (relative error $=10.1 \%$ ) and $27.1 \mu \mathrm{m}$ (relative error $=5.2 \%$ ) for the Paper Mulberry and the Bermuda Grass pollen. respectively. Although SdFFF tends to yield more accurate size distribution due to lower band broadening under the field strength higher than $I G$. the sizes determined by GrFFF were not significantly different from those by SdFFF.
\end{abstract}

Key Words : Gravitational field-flow fractionation (GrFFF), Pollen. Size-characterization

\section{Introduction}

Pollen is one of the most common causes of human allergic reactions. ${ }^{1-3}$ Tiny pollen grains are released from various plants (trees. weeds. and grasses), and enter human respiratory systems. triggering a type of seasonal allergic rhinitis called pollen allergy' better known as "hay fever". Although many of the foods. medicines. or animals that cause allergies can be avoided it is not easy to avoid pollens. Even staying indoors may not help when the pollen count is high.

Since it was first reported ${ }^{3}$ the pollen allergy has been studied by many researchers. It has been reported that the size distributions of pollens are usually broad and the size distribution is one of important factors to the allergic symptoms. ${ }^{1}$ Approximately $20 \%$ of population in industrialized countries suffers from IgE-mediated (Ty'pe I) allergic diseases such as bronchial astluma. conjunctivitis and rhinitis. ${ }^{4}$ In Republic of Korea. about $30 \%$ of adult showed positive reaction to skin-test for pollen allergy. ${ }^{2}$

Field-flow fractionation (FFF) is a family of separation tecluniques which have been demonstrated to be able to separate and characterize high molecular weight species in a size range spanuing five orders of magnitude. from macromolecules to micron-sized particles. ${ }^{5-7}$ Among the members of FFF, gravitational field-flow fractionation (GrFFF) is a teclunique that has been shown to be suitable for quick separation and characterization of particles of about 1-100 $\mu \mathrm{m}$ in size ${ }^{\delta}$ Like other FFF members. GrFFF is a chromatography-like separation teclunique that uses an external field (or gradient). In GrFFF the external field is the earth's gravity which is applied perpendicularly to a thin (about $100-200 \mu \mathrm{m}$ thick), empty channel having rectangular crosssection. There is no packing material (stationary phase) inside the channel and the sample is swept down the channel by the carrier liquid. Particles are driven towards the channel outlet at different velocities depending on their sizes. GrFFF has been employed for separation of various particulate materials including red blood cells. ${ }^{9-11}$ parasites. ${ }^{12.13}$ wine yeast. ${ }^{14.15}$ wheat starch. ${ }^{16.17}$ inorganic particles. ${ }^{18-21}$ and particles of biological interest. $2-2-4$

The aim of this study is to investigate the applicability of GrFFF for separation and the size-characterization of pollens. and then to develop a GrFFF method for size-determine of pollens.

\section{Theory}

In an ideal steric mode of GrFFF. where there is no surface interactions. no friction, no flow rate-induced lift forces. the retention ratio. which is defined by the ratio of the retention time of the carrier $\left(f^{\circ}\right)$ to that of the sample $\left(t_{r}\right), R$ is given by ${ }^{18}$

$$
R=\frac{t^{o}}{t_{r}}=\frac{3 d}{w}
$$

where $d$ is the particle diameter and $w$ is the thickness of the GrFFF channel. According to eqn. (1). $R$ is directly proportional to the particle diameter. and thus GrFFF provides size-based separation. 
In real world, $R$ could be either larger or smaller than $3 d / w$ depending upon the experimental conditions (such as the particle density and diameter. flow rate and the chemical composition of the carrier. etc.). and is expressed by $y^{2 \pm}$

$$
R=\frac{3 \gamma d}{w}
$$

Here $\gamma$ is a "correction factor" adopted to take the dependence of $R$ on experimental conditions into account. In an ideal case. where the particle are moved only by the flow stream at the distance of one particle radius away from the wall. the value of $\gamma$ is 1 , and eqn 2 becomes Eq. (I). Unfortunately $\gamma$ has not yet been completely understood, and thus the size-analysis by GrFFF requires a calibration. ${ }^{26}$

For GrFFF calibration. a series of standards (with known diameters) are injected. and from their observed retention times $\left(t_{\mathrm{T}}\right)$. a plot of $\log t_{\mathrm{r}} v \cdot \log d$ is obtained. Assuming $\gamma$ is a constant this plot is expected to be linear. A first order least square fitting to this plot results in a calibration plot which is generally expressed by ${ }^{27}$

$$
\log t_{r}=-S_{a l} \log d+\log t_{r l}
$$

. where $S_{a}$ is diameter-based selectivity, which is typically around 0.7-0.8 and $t_{r}$ is a constant equal to the extrapolated value of retention time for particles of unit diameter. By using the calibration parameters $S_{a}$ and $t_{r}$. mass-based particle size distribution. $m(d)$, which gives the relative mass as a function of diameter. can be obtained by ${ }^{2 s}$

$$
m(d)=c\left(t_{r}\right) \dot{V}\left|\frac{d t_{r}}{d d}\right|=c\left(t_{r}\right) V S_{d t_{r} 1}\left(\frac{t_{F}}{t_{f}}\right)^{\left.1 s_{d}+1\right) s_{d}}
$$

. where $c(t)$ is the detector signal at retention time $t_{\text {; }}$ and $V$ is the volumetric flow rate. In Eq. (3), if the signal is divided by $\vec{d}$. the mass distribution is converted to the number-based size distribution. ${ }^{\text {sy }}$ Ideally. the densities of the standard and the sample must be same because. if not. $\gamma$ of the standard might be different from that of the sample. resulting in inaccurate size measurement.

\section{Experimental Section}

The pollens used in this study were Paper Mulberry and Bermuda Grass pollen purchased from Duke Scientific Corp. (California. USA). The Paper Mulbeny pollen has the nominal size range of 11-17 $\mu \mathrm{m}$ and the Bermuda Grass pollen 21-29 $\mu \mathrm{m}$. When dry pollens were swelled in water. they are in spherical shape as confurmed by optical microscopy. Their nominal size pertains to the hydrated spherical condition.

The polystyrene latex standards were also purchased from Duke Scientific Corp. and have nominal diameters of 4.5 (with the coefficient of variation (CV) of $20.0 \%$ ). 14.9 (with CV of $14.8 \%$ ). 21.4 (with CV of $15.0 \%$ ). and $45.6 \mu \mathrm{m}$ (with $\mathrm{CV}$ of $14.5 \%$ ) and the density of $1.05 \mathrm{~g} / \mathrm{mL}$.

The GrFFF channel dimensions were $2 \mathrm{~cm}$ in breadth $(b)$. $51 \mathrm{~cm}$ in length $(L)$, and $254 \mu \mathrm{m}$ in thichness (w). GrFFF sy stem was equipped with a Gilson Minuplus 3 Peristaltic
Pump (Gilson Medical Electronics. Middleton. WI. USA) and a Young-Lin M720 UV/VIS detector operating at the wavelength fixed at $254 \mathrm{~cm}$ (Young-Lin Science. An-Yang. Korea). The void volume $\left(V^{n}\right)$ was measured to be $2.8 \mathrm{~mL}$ from the retention volume of $0.5 \%$ acetone $(\mathrm{v} / \mathrm{v})$.

The Sedimentation FFF (SdFFF) channel is $90 \mathrm{~cm}$ in tipto-tip length. $1.5 \mathrm{~cm}$ in breadth, and $0.019 \mathrm{~cm}$ in thickness. The rotor radius is $15.1 \mathrm{~cm}$. The carrier solution was pumped by a M930 HPLC pump (Young-Lin Scientific Co.. Anyang. Korea). The elution of particles was monitored by a UV-106 UV/VIS detector (Linear Instruments. Reno. USA) operating at the wavelength of $254 \mathrm{~nm}$. The SdFFF system was controlled and the data were collected using a computer and the software progranmed in this laboratory. The data processing was performed by the software obtained from Postnova USA (Salt Lake City. Utah. USA). The carrier liquid was water containing $0.1 \%$ (w/v) FL-70 (Fisher Scientific. Fair Lawn. NJ. USA) and $0.02 \%(\mathrm{w} / \mathrm{v})$ sodium azide $\left(\mathrm{NaN}_{3}\right)$ as a bactericide. All experiments were performed at room temperature

\section{Results and Discussion}

Dispersing of pollens. Various aqueous media were tested to select a dispersing liquid for pollens. Figure I shows UV/ VIS absorbance measured at $254 \mathrm{~nm}$ continuously for 10 min for the Paper Mulberry pollens dispersed in various aqueous media. All suspensions show similar trends - the absorbance decreases with time at the beginning. due to settling of the pollens. and after about 4 min reaches a steady state.

Figure 2 shows UV/VIS absorbance measured by the same way for the Paper Mulberry pollen dispersed in water containing $0.1 \% \mathrm{FL}-70$ after sonication. The sonication time was 0.1 .3 . and $5 \mathrm{~min}$. respectively. All suspensions show similar trends in absorbance as in Figure 1 with no significant differences.

Figure 3 shows UV/VIS absorbance measured again by the same way for Paper Mulberry pollen dispersed in water

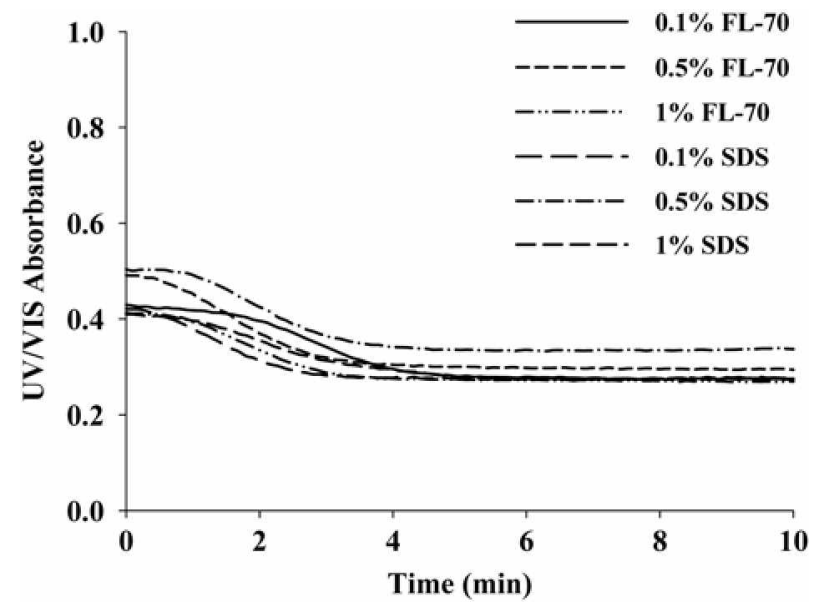

Figure 1. UVVIIS absorbance measured continuously at $254 \mathrm{~nm}$ tor Paper Mulberry pollen dispersed in various aqueous media. 


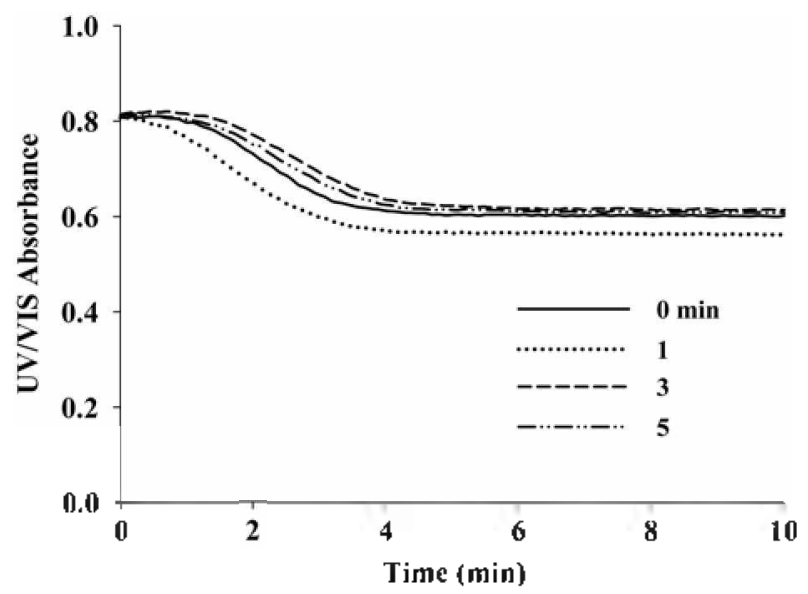

Figure 2. UV/VIS absorbance measured continuously at $254 \mathrm{~nm}$ for Paper Mulberry pollen dispersed in water containing $0.1 \%$ FL70 after sonication. The sonication time was $0.1,3$, and $5 \mathrm{mint}$, respectively:

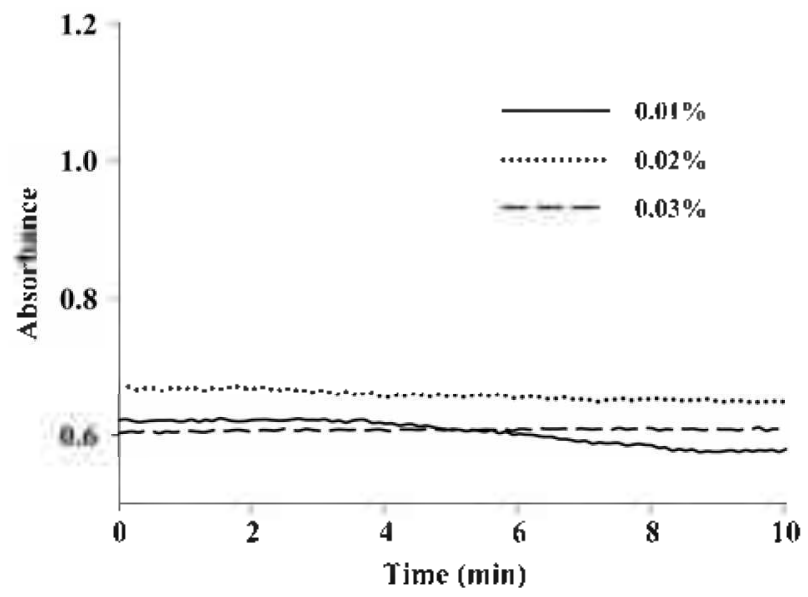

Figure 3. UV/VIS absorbance measured continuously at $254 \mathrm{~nm}$ for Paper Mulberry pollen dispersed in water containing $0.1 \% \mathrm{FL}$ 70 and various concentrations of sodimn azide $\left(\mathrm{NaN}_{3}\right)$.

containing $0.1 \% \mathrm{FL}-70$ and various concentrations of sodium azide $\left(\mathrm{NaN}_{3}\right)$, a bactericide. Agail, no significant differences were found. Based on the data shown in Figure 1-3, water containing $0.1 \%$ FL- 70 and $0.02 \%$ sodium azide was chosen as dispersing medium (and also as the carrier in GrFFF) for pollens and $3 \mathrm{~min}$ as the sonication time in this study.

Optical microscopy analysis of pollens. Figure 4 shows optical microscopy (OM) pictures of the Paper Mulberry and the Bernuda grass pollens dispersed in water containing $0.1 \%$ FL- 70 and $0.02 \%$ sodium azide. Both pollens are in almost spherical shapes. The size distributions of the pollens determined by $\mathrm{OM}$ are shown in Figure 5. It can be seen that both pollens have broad size distributions with the average sizes of 13.8 and $28.6 \mathrm{~mm}$ for the Paper Mulberry and the Bermuda grass pollens. respectively; which are in good agreements with the nominal values.

GrFFF calibration. Figure 6 shows GrFFF fractograms of four polystyrene latex standards (having nominal diameters of $4.5,14.9,21.4$ and $45.6 \mathrm{~mm}$ ) obtained at the flow rate of

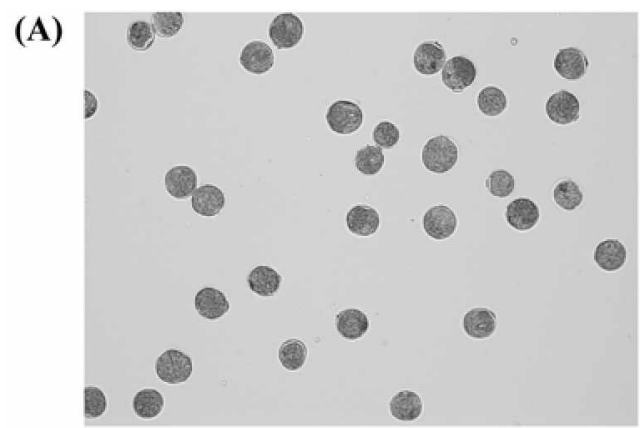

(B)

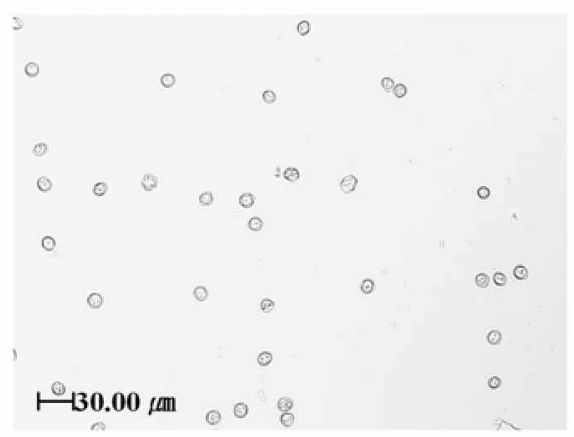

X200

Figure 4. Optical Micrographs (magnification of 200 ) of Bemula grass (A) and Paper Mulberry (B) pollens dispersed in water with $0.1 \% \mathrm{FL}-70$.

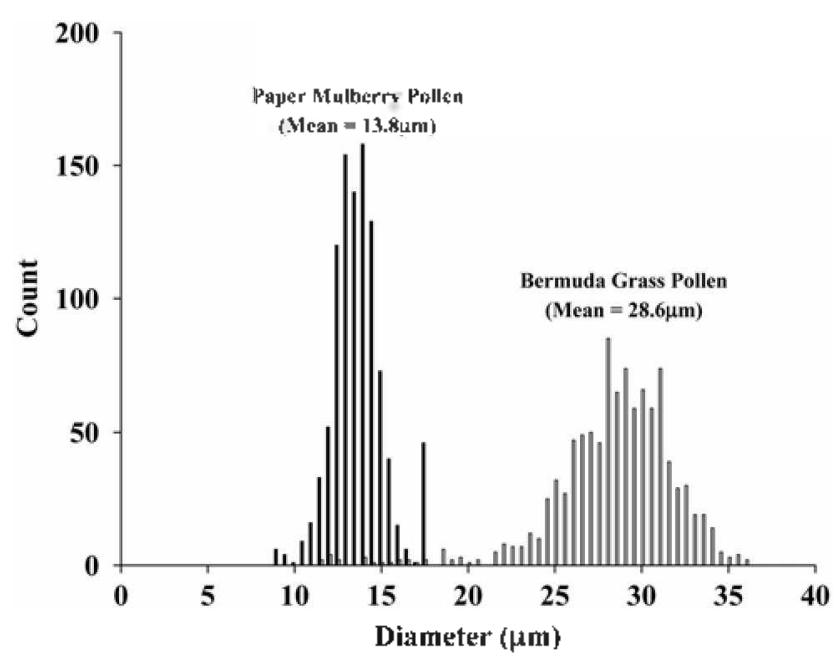

Figure 5. Size distributions determined by OM for Paper Mulberry and the Bermuda Grass pollens.

$2 \mathrm{~mL} / \mathrm{min}$. Their retention data are summarized in Table 1. As shown in Table 1. the values of $\gamma$ is different for particles of different sizes. This will cause the calibration plot deviate from linearity. The value of $\gamma$ increases with size. probably due to increased lyydrodynamic lift forces for larger particles. ${ }^{26,2 y, 3 i}$ Figure 7 shows the calibration plot (plot of $\log t$. v. $\log d$ ) obtained by using the retention data shown in Table I. As expected, the data deviate somewhat from linearity. and when fitted by the first-order least square method. the correlation coefficient was 0.9388 . The calibration curve shown in Figure 7 was used for size determination of pollens in this study: 


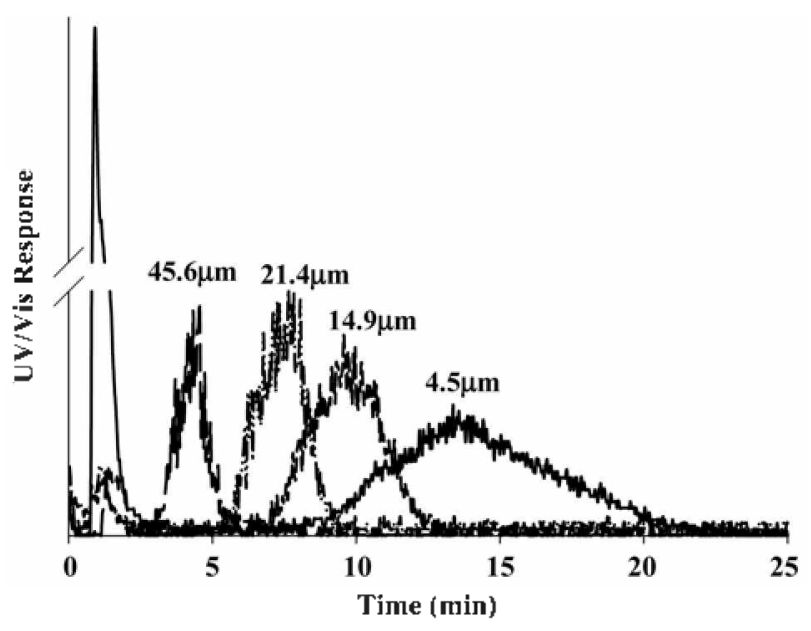

Figure 6. GrFFF fractograms of poly strrene latex standards obtained at flow rate of $2 \mathrm{~mL} / \mathrm{mm}$.

Table 1. Retention data and the steric correction factor ( $y$ ) determmed for polystyrene later standards shown in Figure 6

\begin{tabular}{cccc}
\hline Nominal diameter $(\mu \mathrm{m})$ & $t_{1}(\mathrm{~min})$ & $R$ & $\gamma$ \\
\hline 4.5 & 15.0 & 0.096 & 1.81 \\
14.9 & 9.60 & 0.149 & 0.85 \\
21.4 & 7.10 & 0.202 & 0.80 \\
45.6 & 4.30 & 0.334 & 0.62 \\
\hline
\end{tabular}

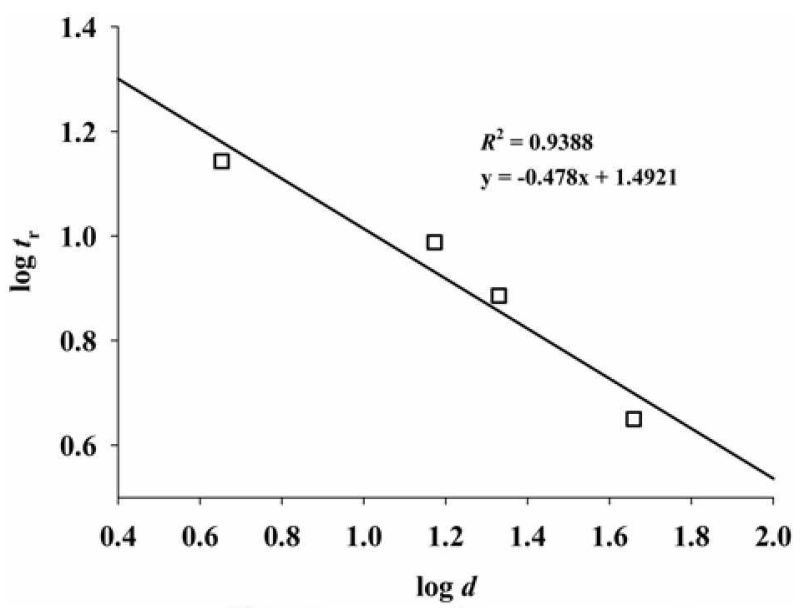

Figure 7. GrFFF calibration curve obtaned from retentıon data of polystyrene latex standards shown in Figure 6.

GrFFF analysis of pollens. Figure 8 shows GrFFF fractograms of the Paper Mulberry and the Bermuda grass pollens obtained at the flow rate of $2 \mathrm{~mL} / \mathrm{min}$. Figure 9 shows the number-based size distributions of the pollens obtained by GrFFF along with those obtained by optical microscopy $(\mathrm{OM})$. For the Paper Mulberry pollen, the mean size obtained by GrFFF is $12.7 \mathrm{~mm}$. and agrees rather well with the $\mathrm{OM}$ data $(13.8 \mu \mathrm{m})$. The relative error is $8.0 \%$. For the Bermuda Grass pollen. the mean size obtained by GrFFF is $32.6 \mathrm{~mm}$. and the relative error is $12.3 \%$. These differences between the results from GrFFF and OM may be attributed to that fact that (1) the calibration curve is not linear. and (2)

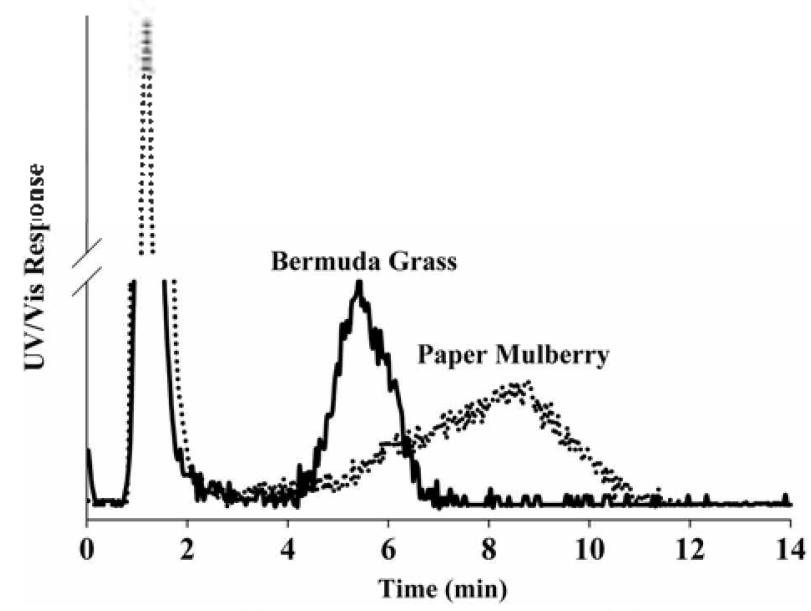

Figure 8. GrFFF tractograms of pollens obtained at the same experimental conditions as in Figure 6 .

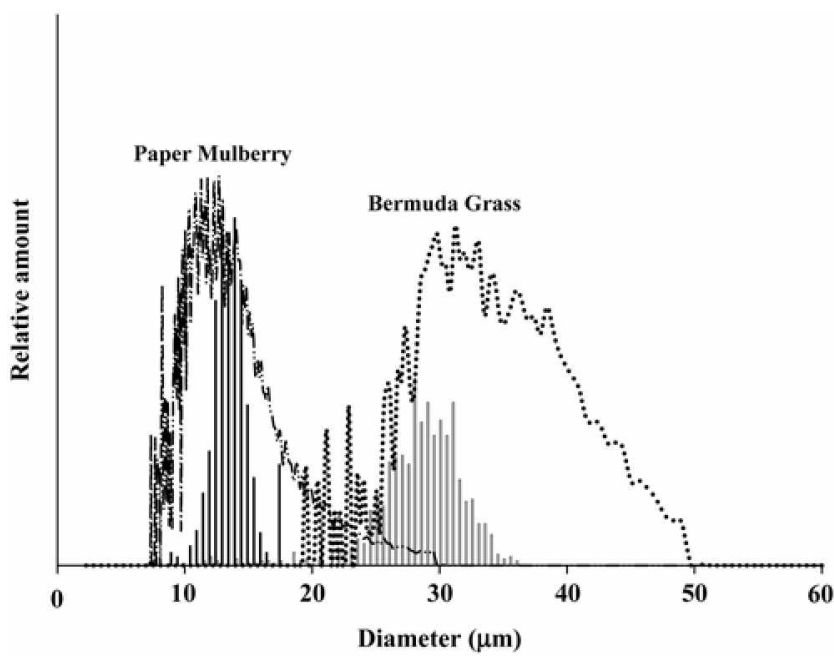

Figure 9. Number-based size distributions of pollens obtained by GrFFF (line) and optical microscopy (bars).

the calibration standards were poly styrene latex beads which are not same as the pollens in chemical composition as well as in physical properties. As shown in Figure 9. the size distributions of both pollens obtained by GrFFF are broader than those from OM, due to band broadening in GrFFF channel during elution.

SdFFF analysis of pollens. One of limitations of GrFFF is that the field strength is not tunable (fixed at 1 gravity). Sedimentation FFF (SdFFF) is another member of FFF. Although the instrumentation and operation of SdFFF are more complicated than those of GrFFF, the separation mechanism of SdFFF is the same as that of GrFFF. The main difference between two teclniques is that. in SdFFF, the field strength can be tuned by adjusting the channel rotation speed.

SdFFF fractograms of the pollens are shown in Figure 10 The channel rotation rate was $400 \mathrm{rpm}$. which is equivalent to 27 gravity. and the flow rate was $3 \mathrm{~mL} / \mathrm{min}$. Figure 11 shows the number-based size distributions of the pollens obtained by SdFFF along with those obtained by optical 


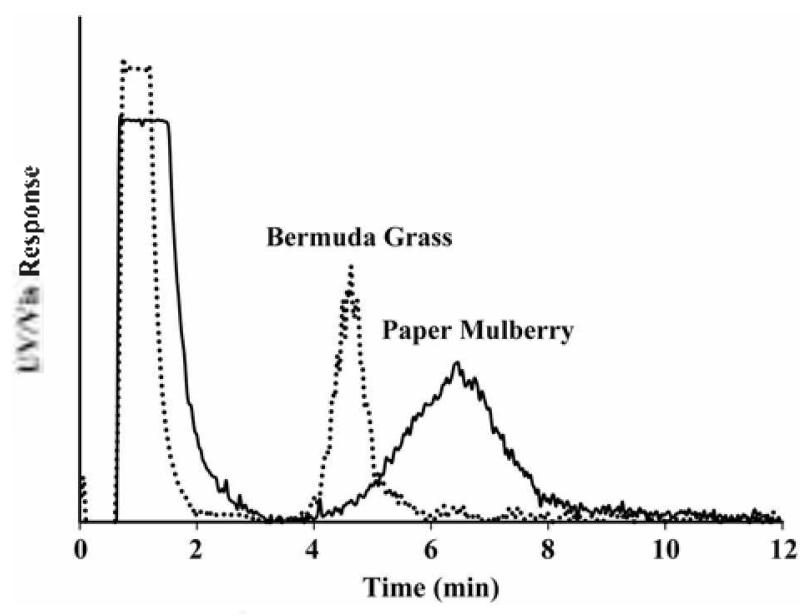

Figure 10. SdFFF fractogranns of pollens obtained at the channel rotation speed of $400 \mathrm{ppm}$ ( 27 gravity) and flow rate of $3 \mathrm{~mL} / \mathrm{min}$.

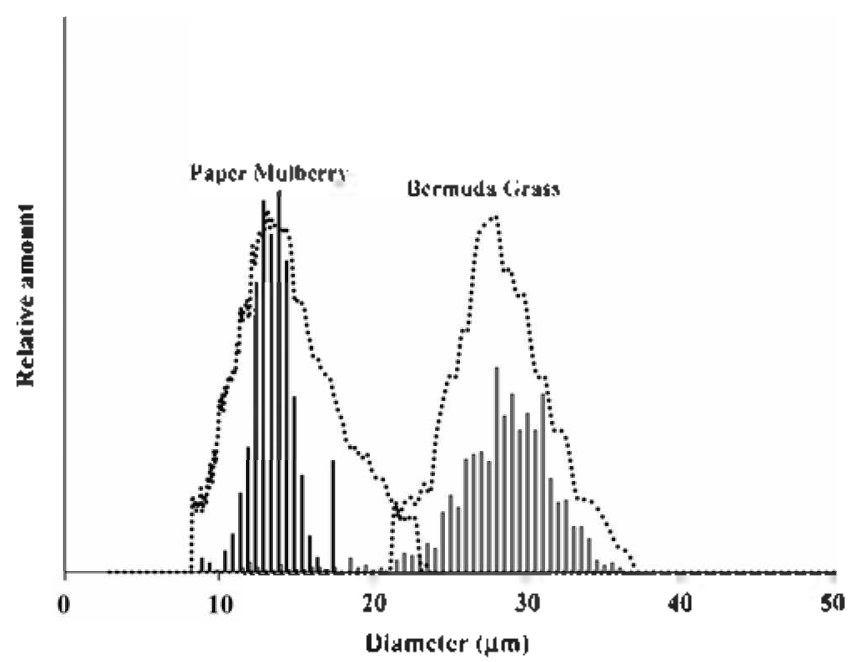

Figure 11. Number-based size distributions of pollens obtained by SdFFF (dotted lines), and by optical microscopy (bars).

microscopy (OM). It can be seen that the band broadening is lower in SdFFF than in GrFFF. especially for the Bemuda Grass pollen. when compared with the data shown in Figure 9.

The mean sizes determined by SdFFF are listed in Table 2. where the mean sizes from GrFFF and OM are also listed. The mean sizes determined by SdFFF are 12.4 (relative error $=10.1 \%$ ) and $27.1 \mu \mathrm{m}$ (relative error $=5.2 \%$ ) for the Paper Mulberry and the Bermuda Grass pollen. respectively. SdFFF yields more accurate sizes for micron-sized pollens in terms of the relative error. probably due to lower band broadening. However the mean sizes from GrFFF and SdFFF are not significantly different from each other.

\section{Conclusion}

GrFFF and SdFFF provide size-based separation of micronsized Paper Mulberry and Bermuda Grass pollens. Both GrFFF and SdFFF yielded the mean sizes that are in reasonable agreements with the results from optical microscopy'
Table 2. Mean diameters measured for pollens by $\mathrm{GrFFF}, \mathrm{OM}$, and SdFFF

\begin{tabular}{ccccc}
\hline \multirow{2}{*}{ Pollen } & \begin{tabular}{c} 
Nominal \\
diameter range \\
\cline { 3 - 5 }
\end{tabular} & \multicolumn{3}{c}{ Mean diameter $(\mu \mathrm{m})$ measured by } \\
\cline { 3 - 5 } & GrFFF & SdFFF & OM \\
\hline Paper Mulbery & $11 \sim 17$ & 12.7 & 12.4 & 13.8 \\
Bemuda grass & $21 \sim 29$ & 32.6 & 27.1 & 28.6 \\
\hline
\end{tabular}

$(\mathrm{OM})$. despite that the calibration standards (polystyrene latex beads) were not same as the pollens in chemical composition as well as in physical properties including the density:

Sedimentation FFF (SdFFF) may yield more accurate size distribution of the pollens due to lower band broadening when high field strength (higher than $1 \mathrm{G}$ ) is employed. The mean sizes obtained by GrFFF are 12.7 (relative enror $=$ $8.0 \%$ ) and $32.6 \mu \mathrm{m}$ (relative error $=12.3 \%$ ). while those by $\mathrm{SdFFF}$ are 12.4 (relative error $=10.1 \%$ ) and $27.1 \mu \mathrm{m}$ (relative error $=5.2 \%$ ) for the Paper Mulberry and the Bermuda Grass pollen. respectively. Still, the mean sizes determined by GrFFF were not significantly different from those by SdFFF

GrFFF is simple in theory. less costly to set up and easier to operate than most other separation techuiques including SdFFF. It is also less time-consuming than OM with total analy sis time of less than about $12 \mathrm{~min}$. With further optimization of GrFFF by reducing the band broadening. GrFFF could be a useful tool for quick size-analy sis of micron-sized pollens and particles of various environmental origins.

Acknowledgements. This work was financially supported by the Korea Research Foundation (R05-2004-000-12516-0) and Hannam University. Dong Young Kang acknowledges the fellowship of the BK2l program from the Ministry of Education and Human Resources Development.

\section{References}

1. Riediker. M.: Koller. T.: Monn. C. Jom of the British Society for Allergy and Chical hmumologv 2000. 6.867.

2. Lee. S.-Y.: Hong. C.-H. The Korea Academy of Pedianic Allergy and Respiratony Disease 1996. 6.92.

3. Noon. L.: Cantab. B. C. The Lancet 1911,1572

4. Liaw, S. H.: Lee, D.-Y; Chow: L. P. Lau. G. X: Su. S. N. Bichlentical and Biophnsical Research Conmtuntcations 2001. 280.738 .

5. Giddings. T. C. Science 1993. 260.1456.

6. Ji. E.; Choi. S.-H.: Yoon, K. R.; Chun. J.-H.; Lee, S. Bull. Korean Chem. Soc. 2006, 27(9), 1433.

7. Kim. H.; Lee, H.; Moon. M. H. Bull. Korean Chem Soc. 2006. $27(3) .413$.

8. Reschiglian. P.: Torsi. G. Chronatophia 1995. 40.467

9. Bernard. A.: Paulet. B.: Colin. V.: Cardot. J. P. Trends in .Anatytical Chemistry 1995. $14(6), 266$.

10. Rasouli. S.: Assidjo. E.: Chianea. T.; Cardot, J. P. J. Chromatogr: 2001. $75 t, 11$.

11. Dugay. A. M.: Cardot. J. P.: Czok. M.: Guernet. M.: Andreux. T. P. J. Chrontatogr: B 1992.579.73.

12. Christian. B.: Cardot. J. P.: Vincent. A.: Christian. P.: Annabelle. M. D.; Bruno, B.: Genevieve. M. J. Chomatog: B 1991, 579, 143.

13. Bernard, A.: Bones. C.: Loiseau. P. M.: Cardot, J. P. J. Chomatog: 
B $1995.66+(2), 444$.

14. Sanz. R.: Puignou. L.: Reschiglian. P.: Galceran. M. T. J. Chromatogr: A 2001.919 .339 .

15. Sanz. R.: Torsello. B.: Reschiglian. P.: Puignou. L.: Galceran. M. T. J. Chromagr. A 2002.996. 135 .

16. Contado, C.: Reschiglian, P: Faccini. S.: Zattoni, A.: Dondi. F. J. Chromatogr: A 2002. 871,449

17. Janouskova. J.: Budinska. M.: Plockova. J: Chmelik. J. J. Chontatogr. \& 2001.914. 183.

18. Giddings. J. C.: Myers. M. N. Sep. Sci. Technol. 1978. 13.637.

19. Giddings. J. C.: Myers M. N.: Caldwell. K. D.: Pav, I. W. J. Chromatogr: 1979, 185.261

20. Caldwell. K. D.: Nguven, T. T.: Myers, M. N.: Giddings, J. C. Sep. Sci. Techol. 1979. 14.935.

21. Pazourek. J.: Chmelik. J. Chromatographia 1993.35. 591.
22. Williams. P. S.; Koch, T; Giddings. J. C. Chem. Eng. Conmun. 1992. H1. 121

23. Yang. F. S.: Caldwell. K. D.: Giddings. J. C. J. Colloid Interface Sci. 1983. 92.81

24. Giddings, J. C.: Myers, M. N.: Caldwell, K. D; Fisher. S. R. Methods of Biochemical thatysis $1980,26.79$.

25. Giddings. J. C. Sep. Sci. Techol. 1978. 13.241.

26. Richard. E. P.: Myers. M. N.: Giddings. J. C. Sep. Sci. Techol. 1984. 19.307.

27. Giddings. T. C.: Moon1. M. H.: Williams. P. S.: Myers. M. N. Anat. Chem 1991, 63. 1366.

28. Moon. M. H; Lee, S. H. J. Mfrocolum Separations 1997, 9(7). 567.

29. Koch. T.: Giddings. T. C. Anal Chent 1986. 58.994.

30. Lee. S. H.: Giddings. T. C. Anal Chent 1988. 60.2328. 